\title{
Metastatic colorectal cancer KRAS genotyping in routine practice: results and pitfalls
}

\author{
Aude Lamy ${ }^{1,2}$, France Blanchard ${ }^{*, 1}$, Florence Le Pessot*,1,3 Richard Sesboüé $^{2}$, \\ Frédéric Di Fiore ${ }^{2,4}$, Jessie Bossut ${ }^{1}$, Elodie Fiant ${ }^{1}$, Thierry Frébourg ${ }^{2,5,6}$ and \\ Jean-Christophe Sabourin ${ }^{1,2,3}$ \\ ${ }^{1}$ Laboratory of Tumor Genetics, University Hospital, Rouen, France; ${ }^{2}$ Inserm U614, Faculty of Medicine, \\ Institute for Medical Research, Rouen University, France; ${ }^{3}$ Department of Pathology, University Hospital, \\ Rouen, France; ${ }^{4}$ Digestive Oncology Unit, Department of Gastroenterology, University Hospital, Rouen, \\ France; ${ }^{5}$ Laboratory of Molecular Genetics, University Hospital, Rouen, France and ${ }^{6}$ Department of Genetics, \\ University Hospital, Rouen, France
}

\begin{abstract}
KRAS genotyping is mandatory before anti-epidermal growth factor receptor monoclonal antibody therapy in metastatic colorectal cancer, which is the second leading cause of cancer-related death in the United States and in Europe. Thus, large-scale KRAS mutation screening is needed for efficient patient management and in the future metastatic colorectal cancer genotyping might also include the detection of the BRAF V600E mutation, which is a very strong negative prognostic factor in colorectal cancer. We report our experience of routine $K R A S / B R A F$ mutation screening practice performed on 1130 formalin-fixed paraffin-embedded tumor samples from 992 colorectal cancer patients. DNA was extracted from macrodissected tumor areas highlighted by a

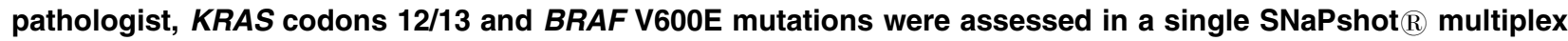
assay and each mutation was confirmed by an independent analysis. KRAS and BRAF mutations were, respectively, present in 41.8 and $6.5 \%$ of the tumor samples. If KRAS and BRAF mutations were mutually exclusive, four samples presented two concomitant KRAS mutations. Genotyping of paired primary tumors and metastases from 44 patients indicated that 5 patients (11.4\%) presented discordant KRAS mutational status. KRAS genotype heterogeneity was also observed within primary tumor sites in seven cases. Non-reproducible KRAS artefactual mutations were detected in 53 samples (4.7\%). We found that the prominent mechanism leading to these artefactual mutations was the fragmentation of DNA occurring during tissue processing. Routine KRAS genotyping performed on formalin-fixed paraffin-embedded tissues requires, therefore, the development of quality control scheme for molecular pathology, especially because of DNA damages induced by formalin fixation. The tumor heterogeneity observed in some patients indicates that it should be more appropriate to perform KRAS genotyping on metastases if sample is available.
\end{abstract}

Modern Pathology (2011) 24, 1090-1100; doi:10.1038/modpathol.2011.60; published online 22 April 2011

Keywords: BRAF; cetuximab; colorectal cancer; formalin-fixed paraffin-embedded; KRAS; mutation; SNaPshot

Oncogenic activation of the epidermal growth factor receptor (EGFR) pathway, commonly observed in cancers, provides survival advantages to neoplastic cells in terms of proliferation, motility, and resistance to apoptosis. ${ }^{1}$ Anti-EGFR therapies have been

Correspondence: Dr A Lamy, PhD, Laboratoire de Génétique Somatique des Tumeurs, CHU de Rouen, Pavillon Jacques Delarue, 1 rue de Germont, 76031 Rouen Cedex, France.

E-mail: aude.lamy@chu-rouen.fr

${ }^{*}$ These authors equally contributed to this work.

Received 29 September 2010; revised 11 January 2011; accepted 11 January 2011; published online 22 April 2011 developed to specifically target this oncogenic cascade, especially in the treatment of metastatic colorectal tumors. Two classes of anti-EGFR agents are available: monoclonal antibodies directed against the extracellular domain of the EGFR and small inhibitory molecules able to inhibit the binding of ATP to the EGFR tyrosine kinase catalytic domain. The FDA and the EMEA have approved the use of anti-EGFR monoclonal antibodies, cetuximab, and panitumumab, in patients with metastatic colorectal carcinoma.

The KRAS protein, belonging to the large superfamily of guanine guanosine-5' $5^{\prime}$ triphosphate (GTP) 
and guanine guanosine-5'-diphosphate (GDP)-binding proteins, is a powerful downstream effector in the EGFR transduction cascade. Somatic KRAS mutations, which are detected in $40 \%$ of colorectal cancer and result in abnormal affinity of KRAS for GTP and permanent activation of the transduction cascade, have been identified as a reliable strong negative predictive factor to anti-EGFR monoclonal antibodies therapies in metastatic colorectal cancer patients. $^{2-4}$ BRAF is located downstream to KRAS and upstream to the MEK-MAP kinases, and the oncogenic BRAF p.V600E mutation, detected in $\sim 5-10 \%$ of colorectal cancer, also results in an activation of the transduction cascade. ${ }^{5}$ Controversial data have been published regarding the predictive value of the $B R A F$ p.V600E mutation in anti-EGFR monoclonal antibodies therapy, but there is a consensus concerning the value of this mutation as a very strong negative prognostic factor in colorectal cancer. ${ }^{6-8}$

Since July 2008, KRAS mutational screening has become mandatory in Europe in order to restrict the treatment of metastatic colorectal cancers with cetuximab and panitumumab to patients with wild-type KRAS tumors and it is likely that, in the future, prescription of anti-EGFR monoclonal antibodies will also require $B R A F$ analysis. ${ }^{9,10}$ Before the integration of KRAS genotyping as a medical analysis, KRAS mutation screening was performed in the context of experimental settings or as part of clinical trials but not in daily routine practice. In this context, the patient cohorts were fairly homogeneous in terms of tissue type, tissue processing, and storage conditions, whereas in a routine setting, tissue sampling, processing, and storage are usually not homogenous. Furthermore, there is at present time a lack of standardization of methods currently used to analyse KRAS in tumors. Considering the medical consequences of KRAS genotyping and the need to ensure high quality and reproducible analyses, technical guidelines for KRAS mutational analyses have been proposed by the European Society of Pathology and the College of American Pathologists. ${ }^{11,12}$ These recommendations emphasized the key role of the pathologist in the selection and preparation of tissue samples, KRAS assay selection, and standardized reporting of results. One of the key points is the tissue on which molecular analyses are performed in the pathology laboratory. Indeed, molecular analyses are generally performed on formalin-fixed paraffin-embedded specimens derived either from the primary tumor or from metastatic sites. Formalin fixation and paraffin embedding processing has been shown to alter DNA. ${ }^{13,14}$ However, only very little data regarding DNA alteration and KRAS genotyping have been reported.

We present here our experience on $>1000$ formalin-fixed paraffin-embedded tumor samples of KRAS/BRAF mutation screening practice, based on a single, fast, and reliable assay using the
SNaPshot $\AA$ technology ${ }^{15}$ and discuss our results, especially discordant cases, in order to avoid such pitfalls and to propose new quality control schemes.

\section{Materials and methods}

\section{Samples}

A total of 1130 formalin-fixed paraffin-embedded tumor samples from 992 colorectal cancer patients were referred for KRAS genotyping to our laboratory between September 2006 and December 2009. Serial sections were cut from each paraffin block and placed on glass slides: the first $3 \mu$ m-thick section was stained with hematoxylin \& eosin (H\&E) for histopathological examination and the five following $10 \mu \mathrm{m}$-thick sections were processed for tumor DNA preparation. The microtome razor blade was changed between each formalin-fixed paraffin-embedded tumor sample and the paraffin sections were processed individually to avoid cross-contamination. H\&E preparation enabled tumor area delimitation and tumor cell percentage visual estimation. To eliminate non-malignant, stromal, and contaminating inflammatory cells and to enrich the analyzed specimen with tumor cells, tumor areas highlighted by a pathologist on H\&E preparation were macrodissected on each of the five $10 \mu \mathrm{m}$-thick sections placed on glass slides using a single-use sterilized scalpel.

\section{Molecular Analyses}

Paraffin macrodissected samples were then placed in sterile tubes. After deparaffinization and rehydration, DNA was isolated using the RecoverAll ${ }^{\mathrm{TM}}$ Total Nucleic Acid Isolation Kit for FFPE Tissues (Applied Biosystems, Courtaboeuf, France). KRAS exon 2 and $B R A F$ exon 15 were simultaneously PCR amplified using the following primers: $5^{\prime}$-AAGG CCTGCTGAAAATGACTG-3' (KRAS-F), 5'-CAAAGA ATGGTCCTGCACCAG-3' (KRAS-R), 5'-CATAATGC TTGCTCTGATAGG-3' (BRAF-F), and 5'-GACTTTCT AGTAACTCAGCAGC-3' (BRAF-R). PCR was performed in a $25 \mu \mathrm{l}$ volume, containing 100-1000 ng of genomic DNA, $1 \times$ Qiagen multiplex PCR master mix (Qiagen, Courtaboeuf, France) and $0.4 \mu \mathrm{M}$ of each primer. After DNA denaturation and polymerase activation steps of $15 \mathrm{~min}$ at $95^{\circ} \mathrm{C}$, the PCR consisted of 45 cycles of $30 \mathrm{~s}$ at $94^{\circ} \mathrm{C}, 90 \mathrm{~s}$ at $60^{\circ} \mathrm{C}$, and $90 \mathrm{~s}$ at $72^{\circ} \mathrm{C}$, and was followed by a final extension step of $10 \mathrm{~min}$ at $72^{\circ} \mathrm{C}$. After purification of the PCR products using the NucleoSpin $\AA$ Extract II kit (Macherey-Nagel EURL, Hoerdt, France), genotyping was performed using the ABI PRISM SNaPshot $\mathbb{R}$ Multiplex Kit (Applied Biosystems). We designed, for each DNA strand, five SNaPshot $\AA$ primers, complementary in their $3^{\prime}$-end to KRAS с. $34 \mathrm{G}$, с. $35 \mathrm{G}$, с. $37 \mathrm{G}$, с.38G and $B R A F$ с. $1799 \mathrm{~T}$ adjacent nucleotides and containing $5^{\prime}$-end 
Table 1 KRAS and BRAF SNaPshot $®$ primer characteristics: sequences, extended nucleotides and concentrations

\begin{tabular}{|c|c|c|c|c|c|}
\hline \multirow[t]{2}{*}{ Extended nucleotide position } & \multirow[t]{2}{*}{ Sequence } & \multirow[t]{2}{*}{ Strand } & \multicolumn{2}{|c|}{ Primer extension } & \multirow{2}{*}{$\begin{array}{c}\text { Concentration } \\
\mu M\end{array}$} \\
\hline & & & Wild type & Mutant & \\
\hline KRAS с.34 & $\begin{array}{l}\text { 5'-AACTTGTGGTAGTTGGAGCT-3' } \\
\text { 5'-GGCACTCTTGCCTACGCCAC-3' }\end{array}$ & $\begin{array}{l}\text { Sense } \\
\text { Antisense }\end{array}$ & $\begin{array}{l}\mathrm{G} \\
\mathrm{C}\end{array}$ & $\begin{array}{l}\mathrm{C} / \mathrm{A} / \mathrm{T} \\
\mathrm{G} / \mathrm{T} / \mathrm{A}\end{array}$ & $\begin{array}{l}0.02 \\
0.06\end{array}$ \\
\hline KRAS с.35 & $\begin{array}{l}5^{\prime}-\mathrm{N}_{10} \text { ACTTGTGGTAGTTGGAGCTG-3' } \\
5^{\prime}-\mathrm{N}_{10} \text { AGGCACTCTTGCCTACGCCA- } 3^{\prime}\end{array}$ & $\begin{array}{l}\text { Sense } \\
\text { Antisense }\end{array}$ & $\begin{array}{l}\mathrm{G} \\
\mathrm{C}\end{array}$ & $\begin{array}{l}\mathrm{C} / \mathrm{A} / \mathrm{T} \\
\mathrm{G} / \mathrm{T} / \mathrm{A}\end{array}$ & $\begin{array}{l}0.015 \\
0.12\end{array}$ \\
\hline KRAS с.37 & $\begin{array}{l}5^{\prime}-\mathrm{N}_{20} \text { TTGTGGTAGTTGGAGCTGGT-3' } \\
5^{\prime}-\mathrm{N}_{20} \text { CAAGGCACTCTTGCCTACGC-3' }\end{array}$ & $\begin{array}{l}\text { Sense } \\
\text { Antisense }\end{array}$ & $\begin{array}{l}\mathrm{G} \\
\mathrm{C}\end{array}$ & $\begin{array}{l}\mathrm{C} / \mathrm{A} / \mathrm{T} \\
\mathrm{G} / \mathrm{T} / \mathrm{A}\end{array}$ & $\begin{array}{l}0.04 \\
0.16\end{array}$ \\
\hline KRAS с.38 & $\begin{array}{l}5^{\prime}-\mathrm{N}_{30} \text { TGTGGTAGTTGGAGCTGGTG-3' } \\
5^{\prime}-\mathrm{N}_{30} \text { TCAAGGCACTCTTGCCTACG-3' }\end{array}$ & $\begin{array}{l}\text { Sense } \\
\text { Antisense }\end{array}$ & $\begin{array}{l}\mathrm{G} \\
\mathrm{C}\end{array}$ & $\begin{array}{l}\mathrm{C} / \mathrm{A} / \mathrm{T} \\
\mathrm{G} / \mathrm{T} / \mathrm{A}\end{array}$ & $\begin{array}{l}0.02 \\
0.2\end{array}$ \\
\hline$B R A F$ c.1799 & $\begin{array}{l}5^{\prime}-\mathrm{N}_{40} \text { GGTGATTTTGGTCTAGCTACAG-3' } \\
5^{\prime}-\mathrm{N}_{40} \text { ACCCACTCCATCGAGATTTC- } 3^{\prime}\end{array}$ & $\begin{array}{l}\text { Sense } \\
\text { Antisense }\end{array}$ & $\begin{array}{l}\mathrm{T} \\
\mathrm{A}\end{array}$ & $\begin{array}{l}\mathrm{A} \\
\mathrm{T}\end{array}$ & $\begin{array}{l}0.08 \\
0.01\end{array}$ \\
\hline
\end{tabular}

additional tails of various sizes, which allowed their simultaneous detection. Two multiplex SNaPshot $\mathbb{R}$ reactions were performed in a final $10 \mu \mathrm{l}$ volume, containing $2 \mu \mathrm{l}$ of PCR product, $2.5 \mu \mathrm{l}$ of Ready Reaction Mix with fluorescent dideoxynucleotides, $1 \mu \mathrm{l}$ of sequencing buffer (from Big Dye ®) Terminator v3.1 cycle sequencing kit, Applied Biosystems), and KRAS/BRAF sense or antisense $\mathrm{SNaPshot}{ }^{\circledR}$ primers (see Table 1 for concentrations). The reaction consisted of 25 cycles of $10 \mathrm{~s}$ at $96^{\circ} \mathrm{C}, 5 \mathrm{~s}$ at $50^{\circ} \mathrm{C}$, and $30 \mathrm{~s}$ at $60^{\circ} \mathrm{C}$ (rapid thermal ramps were used). $\mathrm{SNaPshot} \AA$ products were then treated with three units of Shrimp Alkaline Phosphatase (GE Healthcare, Orsay, France) and incubated for $1 \mathrm{~h}$ at $37^{\circ} \mathrm{C}$ to remove unincorporated dideoxynucleotide triphosphates. After enzyme inactivation for $15 \mathrm{~min}$ at $75^{\circ} \mathrm{C}$, labelled products were separated on $36 \mathrm{~cm}$-long capillaries in POP7 polymer during $25 \mathrm{~min}$ in an automated sequencer (ABI PRISM 3130xl Genetic Analyzer, Applied Biosystems). Data analyses were performed using GeneMapper Software version 4.0 (Applied Biosystems).

\section{UNG Treatment}

DNA (500 ng) was treated with Escherichia coli uracil N-glycosylase (UNG, Roche Diagnostics, Meylan, France) before amplification. A control experiment was performed by amplifying the same amount of non-treated DNA.

\section{Quality Control}

Five controls were included to assess the specificity of the KRAS/BRAF SNaPshot $\AA$ assay in each batch: mutant $K R A S$, mutant $B R A F$, wild-type KRAS/BRAF DNAs, and two controls with no DNA. Controls were handled from amplification to SNaPshot ${ }^{\circledR}$ results interpretation. Each detected mutation led to a second independent analysis performed on a second DNA extraction. The paraffin block was sectioned independently to avoid contamination with tumor tissue from another patient sectioned in the same series. If a discrepancy was observed between the two analyses, a third amplification from a third DNA extraction was performed. In the case of discrepancy between the three analyses, the mutations detected were considered as artefactual and the analyses were considered as non-contributive. Figure 1 summarizes our quality approach.

\section{Results}

\section{KRAS and BRAF Genotyping Assay}

Our KRAS/BRAF assay simultaneously explores five nucleotides for the 13 possible reported point mutations. Figure 2 illustrates the assay performed on formalin-fixed paraffin-embedded extracted DNAs containing a KRAS and a BRAF mutation, respectively. The specificity, sensitivity, and reproducibility of our assay were evaluated for the seven reported deleterious KRAS mutations in quality control schemes. Serial dilutions (from 100 to $5 \%$ ) of wild-type vs mutant cell lines extracted DNA were assessed in triplicate. The sensitivity was $5 \%$ for KRASG12C, KRASG12D, KRASG12A, KRASG12V, and KRASG13D; between 5 and 25\% for KRASG12R; 25\% for KRASG12S. In the light of these results and to avoid false negative results, we considered that a minimum of $20 \%$ of tumor cells should be present in the sample to perform KRAS/ $B R A F$ genotyping.

\section{Spectrum of KRAS and BRAF Mutations in Colorectal Cancers}

In our 1130 specimens series, KRAS mutational status was successfully assessed in 1077 formalinfixed paraffin-embedded tumor samples obtained from primary (916 cases) or metastatic (161 cases) sites originating from 951 patients with colorectal cancers. Mutations within codons 12 and 13 were detected in $450(41.8 \%)$ of the analyzed tumor samples. The three main mutations found were c.35G > A (159 mutated tumor samples, 35.3\%), 


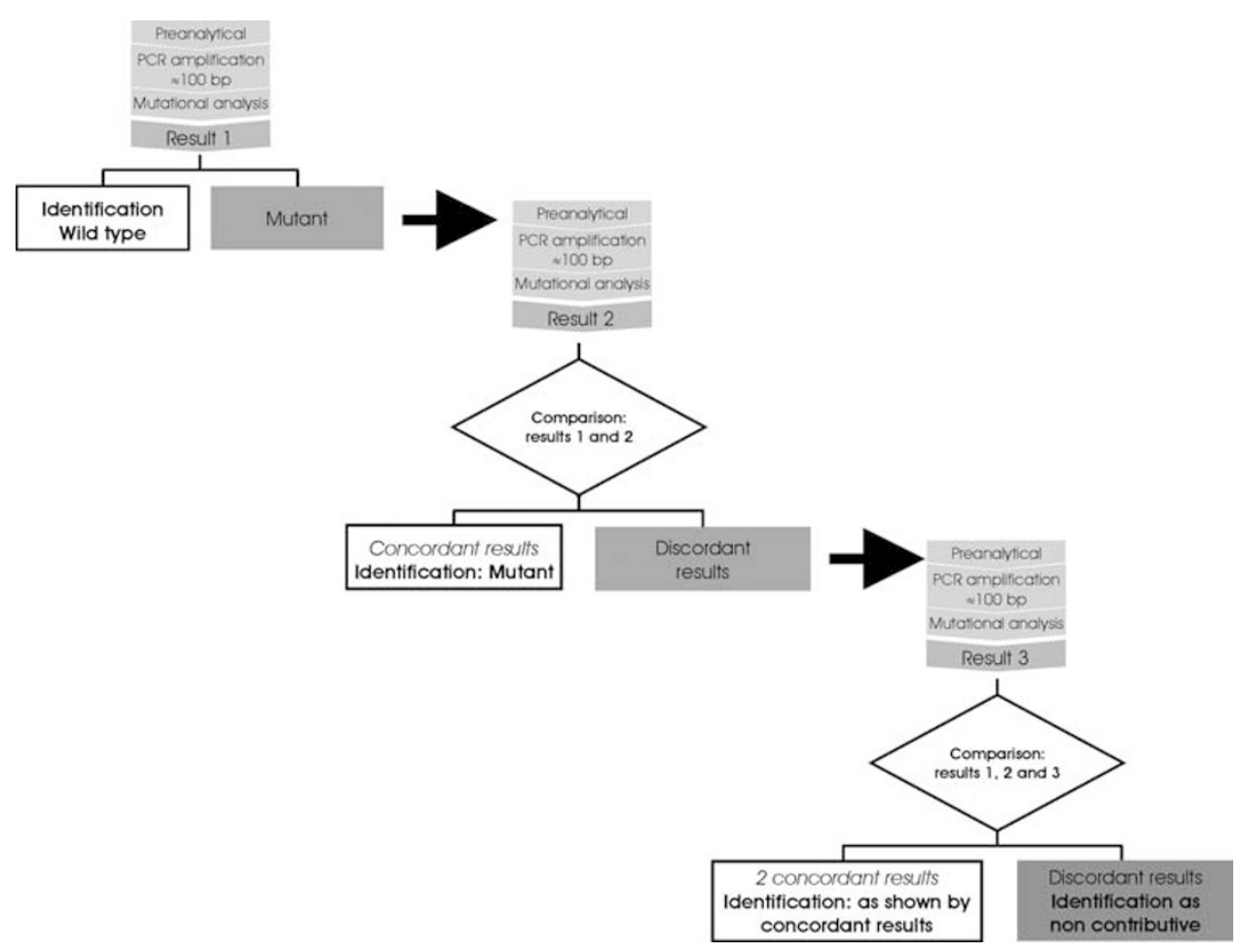

Figure 1 Scheme of quality approach in KRAS/BRAF genotyping.

c.38G $>$ A (99 mutated tumor samples, $22.0 \%$ ), and c.35G $>\mathrm{T}$ (91 mutated tumor samples, 20.2\%), which accounted for $77.5 \%$ of all the mutated cases (Table 2). This pattern was observed for both primary tumors and metastases, but a slight difference was observed in the distribution: the predominant type was c.35G $>$ A $(37.5 \%)$ for primary tumors and both c.35G $>\mathrm{T}$ and c.38G $>\mathrm{A}(29.5 \%)$ for metastatic sites. The vast majority of KRAS mutations are variations of a single nucleotide in codon 12 or 13. Four formalin-fixed paraffin-embedded tumor samples presented with double KRAS mutations (ie, a mutation with two wrong nucleotides): one liver metastasis presented a double KRAS c. $34 \mathrm{G}>\mathrm{C} / \mathrm{c} .34 \mathrm{G}>\mathrm{T}$ mutation, a colon primary tumor showed a double KRAS c.38G $>$ T/c.39C $>\mathrm{T}$ mutation, and two colon primary tumors harbored a double KRAS c.34G > T/c.35G > T mutation. In the latter two cases, mutations occurred on the same allele as deduced from the $\mathrm{SNaPshot} \AA$ pattern: KRAS c.34G $>\mathrm{T}$ and c.35G $>\mathrm{T}$ mutations were detected using only the forward and the reverse SNaPshot $\AA$ primers, respectively, suggesting that the c.35G forward primer and the c.34G reverse primer were not extended from the mutated allele due to $3^{\prime}$ mismatch.

Because of new release data on $B R A F$ implication in colorectal carcinogenesis and anti-EGFR therapy, $B R A F$ mutational status was successfully assessed with KRAS in a subset of the patients: ${ }^{6-8}$ among the 397 cases tested, 26 tumor samples $(6.5 \%)$ presented the c.1799T $>$ A, p.V600E BRAF mutation.
As expected, KRAS and BRAF mutations were found to be mutually exclusive. ${ }^{16}$

\section{KRAS Mutation Variation Status According to the Tumor Sites}

In this series, 70 primary tumors and their corresponding lymph-node or distant metastases obtained from 44 colorectal cancer patients were analyzed. No discordance was noted in the BRAF analyses. In contrast, discordant results concerning the KRAS mutational status were found in five patients $(11.4 \%)$. In three of them, the mutation was restricted to the primary tumor site whereas the metastasis sites disclosed a wild-type phenotype; in the other two patients, the mutant KRAS was found only in the distant metastasis (Table 3). In one patient (patient 72, Table 3) with a mutant KRAS primary tumor paired with a wild-type KRAS lymph-node metastasis, additional tumor samples were analyzed: the c.38G > A KRAS mutation previously identified in the primary tumor was finally also detected in an additional lymph-node metastasis and in the distant liver metastasis.

Variation in KRAS mutational status was also observed within the primary tumor site in seven cases corresponding to multifocal synchronous tumor localizations (Table 3). Thus, in five patients, KRAS mutations were restricted to one tumor localization with no possibility of metastasis assessment. For one patient, two colon tumor sites 
presented a distinct KRAS mutation type (patient 903, Table 3). Finally, one patient (patient 98, Table 3) presented a pT2No mutated KRAS
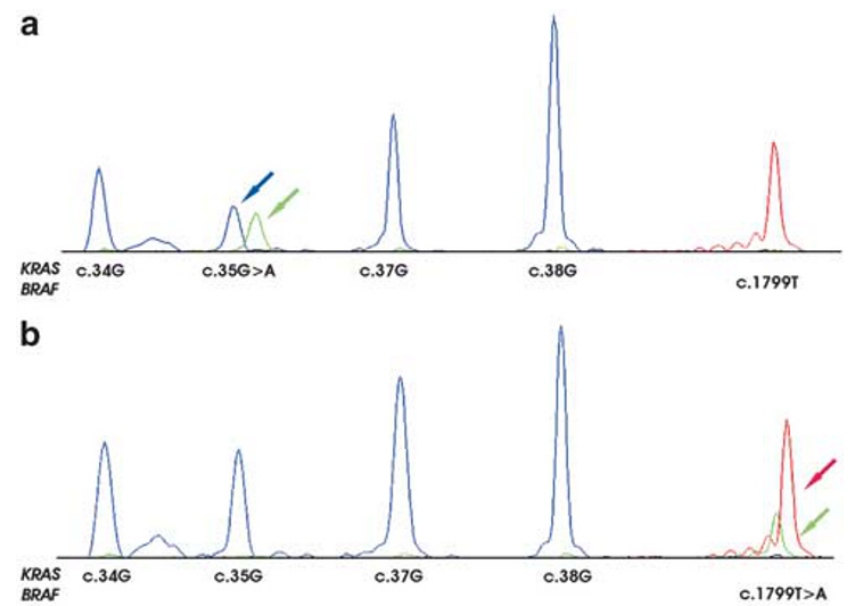

C

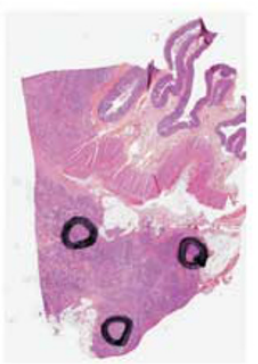

Figure $2 K R A S / B R A F$ genotyping assay. (a) SNaPshot $\AA$ sense pattern obtained from a $K R A S$ c. $35 \mathrm{G}>\mathrm{A}$ mutated/BRAF wild-type formalin-fixed paraffin-embedded extracted DNA. Two peaks were detected for the KRAS c.35 position: one corresponded to the wild-type nucleotide (blue arrow) and the other to the A mutated nucleotide (green arrow). For KRAS c.34, c.37, c.38 and $B R A F$ c.1799 positions, a single peak corresponding to the wildtype nucleotide was detected. (b) SNaPshot $\AA$ sense pattern obtained from a KRAS wild-type/BRAF c.1799T $>$ A formalinfixed paraffin-embedded extracted DNA. Two peaks were detected for the BRAF c.1799 position: one corresponded to the wild-type nucleotide (red arrow) and the other to the A mutated nucleotide (green arrow). For KRAS c.34, c.35, c.37, and c.38, a single peak corresponding to the wild-type nucleotide was detected. (c) Pre-analytical phase: tumor areas are highlighted by a pathologist. More than $80 \%$ of tumor cells are present in these marked zones; colon carcinoma, H\&E staining.
(c.34G $>$ T) rectal tumor and a pT3No wild-type $K R A S$ colon tumor. As expected, considering the staging of these tumors, the liver metastasis investigated 2 years later in this patient was found to derive from the pT3No colon tumor, according to its wild-type KRAS genotype.

\section{Artefactual Mutations}

Among the 397 samples analyzed for $B R A F, 19$ analyses generated irreproducible results that were therefore considered artefactual. These irreproducible results corresponded to the c.1799T $>\mathrm{A} B R A F$ V600E mutation, whereas $\mathrm{T}>\mathrm{C}$ and $\mathrm{T}>\mathrm{G}$ variations were never observed. These $B R A F$ artefactual mutations were found associated with KRAS artefactual mutations in a small subset of tumor samples.

KRAS artefactual mutations were detected in 53 formalin-fixed paraffin-embedded tumor samples obtained from 41 colorectal cancer patients. For each sample, a mean of 4.6 analyses (range from 2 to 13) were performed from independent DNA extraction or amplification and, in each batch of tests, negative and positive controls were valid. In these cases, discordant KRAS mutational patterns between all analyses performed were observed (Figure 3). As a whole, 283 KRAS artefactual mutations were recorded from 187 analyses: 148 $(52.3 \%)$ corresponded to $\mathrm{G}>\mathrm{A}$ transitions, 103 $(36.4 \%)$ to $\mathrm{G}>\mathrm{T}$ transversions, and $32(11.3 \%)$ were $\mathrm{G}>\mathrm{C}$ transversions. Furthermore, 93 analyses $(33 \%)$ presented an abnormal mutational pattern: two or more (up to five) KRAS mutations were detected in the same amplification product and KRAS/BRAF double mutant were present for 16 analyses (Figure $3)$. These KRAS artefactual mutations were preferentially observed for $K R A S$ c.35G (48.4\%), followed by KRAS c.34G (27.6\%) and c.38G $(21.5 \%)$ and were less frequent for KRAS c.37G (2.5\%), (Figure 3). This distribution mimicked that of the observed KRAS somatic mutations. It is noticeable that the intensity of the signal corresponding to the detection of those artefactual mutations was weak, as compared with the expected signal considering the tumor cell

Table 2 Distribution of KRAS and BRAF mutations in colorectal cancer patients, number and (percentage)

\begin{tabular}{|c|c|c|c|c|c|}
\hline Gene & Nucleotide & Protein effect & Primary tumors & Metastasis & Both \\
\hline \multirow[t]{11}{*}{ KRAS } & c. $34 \mathrm{G}>\mathrm{A}$ & p.G12S & $19(4.9 \%)$ & & $19(4.2 \%)$ \\
\hline & c. $34 \mathrm{G}>\mathrm{C}$ & p.G12R & $9(2.3 \%)$ & $1(1.6 \%)$ & $10(2.2 \%)$ \\
\hline & c. $34 \mathrm{G}>\mathrm{T}$ & p.G12C & $33(8.5 \%)$ & $6(9.8 \%)$ & $39(8.7 \%)$ \\
\hline & c. $35 \mathrm{G}>\mathrm{A}$ & p.G12D & $146(37.5 \%)$ & $13(21.3 \%)$ & $159(35.3 \%)$ \\
\hline & c. $35 \mathrm{G}>\mathrm{C}$ & p.G12A & $22(5.7 \%)$ & $4(6.6 \%)$ & $26(5.8 \%)$ \\
\hline & c. $35 \mathrm{G}>\mathrm{T}$ & p.G12V & $73(18.7 \%)$ & $18(29.5 \%)$ & $91(20.2 \%)$ \\
\hline & c. $37 \mathrm{G}>\mathrm{T}$ & p.G13C & $3(0.8 \%)$ & & $3(0.7 \%)$ \\
\hline & c. $38 \mathrm{G}>\mathrm{A}$ & p.G13D & $81(20.8 \%)$ & $18(29.5 \%)$ & $99(22.0 \%)$ \\
\hline & c. $34 \mathrm{G}>\mathrm{C} / 34 \mathrm{G}>\mathrm{T}$ & Unknown & & $1(1.6 \%)$ & $1(0.2 \%)$ \\
\hline & c. $34 \mathrm{G}>\mathrm{T} / 35 \mathrm{G}>\mathrm{T}$ & Unknown & $2(0.5 \%)$ & & $2(0.4 \%)$ \\
\hline & c. $38 \mathrm{G}>\mathrm{T} / 39 \mathrm{C}>\mathrm{T}$ & Unknown & $1(0.3 \%)$ & & $1(0.2 \%)$ \\
\hline$B R A F$ & c. $1799 \mathrm{~T}>\mathrm{A}$ & p.V600E & & & $26(6.5 \%)$ \\
\hline
\end{tabular}


Table 3 Tumor heterogeneity according to KRAS mutational status in colorectal cancer patients

\begin{tabular}{|c|c|c|c|}
\hline Patient no & Event type & Tumor localization & KRAS status \\
\hline 72 & $\begin{array}{l}\text { Primary tumor } \\
\text { Metastasis }\end{array}$ & $\begin{array}{l}\text { Colon } \\
\text { Lymph node }\end{array}$ & $\begin{array}{l}\text { c. } 38 \mathrm{G}>\mathrm{A} \\
\mathrm{WT}\end{array}$ \\
\hline 221 & $\begin{array}{l}\text { Primary tumor } \\
\text { Metastasis }\end{array}$ & $\begin{array}{l}\text { Rectum } \\
\text { Liver }\end{array}$ & $\begin{array}{l}\text { WT } \\
\text { c. } 35 \mathrm{G}>\mathrm{T}\end{array}$ \\
\hline 436 & $\begin{array}{l}\text { Primary tumor } \\
\text { Metastasis }\end{array}$ & $\begin{array}{l}\text { Colon } \\
\text { Lymph node }\end{array}$ & $\begin{array}{l}\text { c. } 35 \mathrm{G}>\mathrm{A} \\
\text { WT }\end{array}$ \\
\hline $512^{\mathrm{a}}$ & $\begin{array}{l}\text { Primary tumor } \\
\text { Synchronous metastasis biopsy }\end{array}$ & $\begin{array}{l}\text { Colon } \\
\text { Liver }\end{array}$ & $\begin{array}{l}\text { WT } \\
\text { c. } 34 \mathrm{G}>\text { C, c. } 34 \mathrm{G}>\mathrm{T}\end{array}$ \\
\hline 89 & $\begin{array}{l}\text { Primary tumor } \\
\text { Metastasis }\end{array}$ & $\begin{array}{l}\text { Colon } \\
\text { Liver }\end{array}$ & $\begin{array}{l}\text { c. } 38 \mathrm{G}>\mathrm{A} \\
\mathrm{WT}\end{array}$ \\
\hline $98^{\mathrm{b}}$ & $\begin{array}{l}\text { T1, pT2N0 primary tumor } \\
\text { T2, pT3N0 primary tumor }\end{array}$ & $\begin{array}{l}\text { Rectum } \\
\text { Colon }\end{array}$ & $\begin{array}{l}\text { c. } 34 \mathrm{G}>\mathrm{T} \\
\text { WT }\end{array}$ \\
\hline 288 & $\begin{array}{l}\text { T1, pT3N0Mx primary tumor } \\
\text { T2, pT2N0Mx primary tumor }\end{array}$ & $\begin{array}{l}\text { Colon } \\
\text { Colon }\end{array}$ & $\begin{array}{l}\text { c. } 35 \mathrm{G}>\mathrm{A} \\
\text { WT }\end{array}$ \\
\hline 541 & $\begin{array}{l}\text { T1, pT3N1 primary tumor } \\
\text { T2, pT3N2 primary tumor }\end{array}$ & $\begin{array}{l}\text { Colon } \\
\text { Rectum }\end{array}$ & $\begin{array}{l}\text { WT } \\
\text { c. } 35 \mathrm{G}>\mathrm{T}\end{array}$ \\
\hline 811 & $\begin{array}{l}\mathrm{T} 1 \text {, primary tumor } \\
\mathrm{T} 2 \text {, primary tumor }\end{array}$ & $\begin{array}{l}\text { Duodenum } \\
\text { Colon }\end{array}$ & $\begin{array}{l}\text { c. } 35 \mathrm{G}>\mathrm{T} \\
\text { WT }\end{array}$ \\
\hline 897 & $\begin{array}{l}\text { T1, primary tumor } \\
\text { T2, primary tumor }\end{array}$ & $\begin{array}{l}\text { Sigmoid } \\
\text { Cecum }\end{array}$ & $\begin{array}{l}\text { WT } \\
\text { c. } 34 \mathrm{G}>\mathrm{T}\end{array}$ \\
\hline 903 & $\begin{array}{l}\text { T1, ypT3N0Mx } \\
\text { T2, ypT3N0Mx }\end{array}$ & $\begin{array}{l}\text { Colon } \\
\text { Colon }\end{array}$ & $\begin{array}{l}\text { c. } 34 \mathrm{G}>\mathrm{T} \\
\text { c. } 35 \mathrm{G}>\mathrm{T}\end{array}$ \\
\hline 90 & $\begin{array}{l}\text { T1, pT3N2Mx primary tumor } \\
\text { T2, pT3N2Mx primary tumor }\end{array}$ & $\begin{array}{l}\text { Colon } \\
\text { Cecum }\end{array}$ & $\begin{array}{l}\text { c. } 35 \mathrm{G}>\mathrm{A} \\
\text { WT }\end{array}$ \\
\hline
\end{tabular}

${ }^{\mathrm{a}}$ The liver metastasis tumorectomy obtained 2 years later was KRAS WT.

${ }^{\mathrm{b}}$ The liver metastasis investigated 2 years later was KRAS WT.

percentage. For five samples, KRAS PCR products were sequenced from antisense primer and artefactual mutations detected by $\mathrm{SNaPshot} \mathbb{R}$ were confirmed (Figure 3).

To test the hypothesis that cytosine deamination resulting in deoxyuridine residues might be responsible for the G>A artefactual transitions that we observed, 16 DNA aliquots were treated with UNG from E. coli. This enzyme removes uracil from DNA, and the resulting abasic site is subsequently hydrolyzed by $\beta$-elimination creating a strand break. ${ }^{17}$ Thus, UNG treatment of the template DNA is expected to lead to the disappearance of the $\mathrm{G}>\mathrm{A}$ transitions if they are due to cytosine deamination. For only one sample presenting a KRAS c.35G $>\mathrm{T}$ transversion (Figure 4), the additional c.35G $>$ A transition disappeared after UNG treatment.

Since molecular jumping events, occurring when degraded templates are PCR amplified, have also been shown to cause substitutions, six DNA samples were PCR amplified using a different set of oligonucleotide primers yielding a shorter KRAS amplicon $(80 \mathrm{bp}) .{ }^{18}$ For one sample presenting multiple non-reproducible KRAS mutations, from three independent DNA extractions performed with the original KRAS $173 \mathrm{bp}$ amplicon, a reproducible KRAS c.35G > A mutation could be evidenced from the KRAS $80 \mathrm{bp}$ PCR amplifications. Furthermore, KRAS $80 \mathrm{bp}$ amplicon analysis helped us to con- clude for 11 samples that showed conflicting results with the original KRAS $173 \mathrm{bp}$ amplicon (Figure 5). These results subsequently prompted us to perform $K R A S$ and BRAF genotyping on shorter amplicons (80 and $126 \mathrm{bp}$, respectively; primer sequences are available upon request).

We observed that a majority of the artefactual mutations were detected in tumor samples obtained from a small subset of pathology laboratories, suggesting that they could have been generated by specific tissue sampling, processing, or storage procedures. Indeed, $66 \%$ of the formalin-fixed paraffin-embedded tumor samples generating noncontributive analyses originated from five laboratories. By questioning the pathologists in charge of these specimens, we learned that the paraffin blocks were stored in high humidity and temperature level $\left(+30^{\circ} \mathrm{C}\right)$. In contrast, no relationship could be established between paraffin block storage time and the prevalence of artefactual mutations (period of storage ranged from 8 to 2263 days (6.2 years) with a mean of 886 days, 2.4 years).

Finally, the use of fresh specimens might resolve these non-contributive analyses. Thus, for one patient with artefactual results after six analyses from two formalin-fixed paraffin-embedded primary tumor samples, a biopsy of a liver metastasis was performed and addressed to our institution in nonformalin reagent (RNAlater $(\mathbb{R})$; this new sample disclosed a wild-type KRAS genotype. 


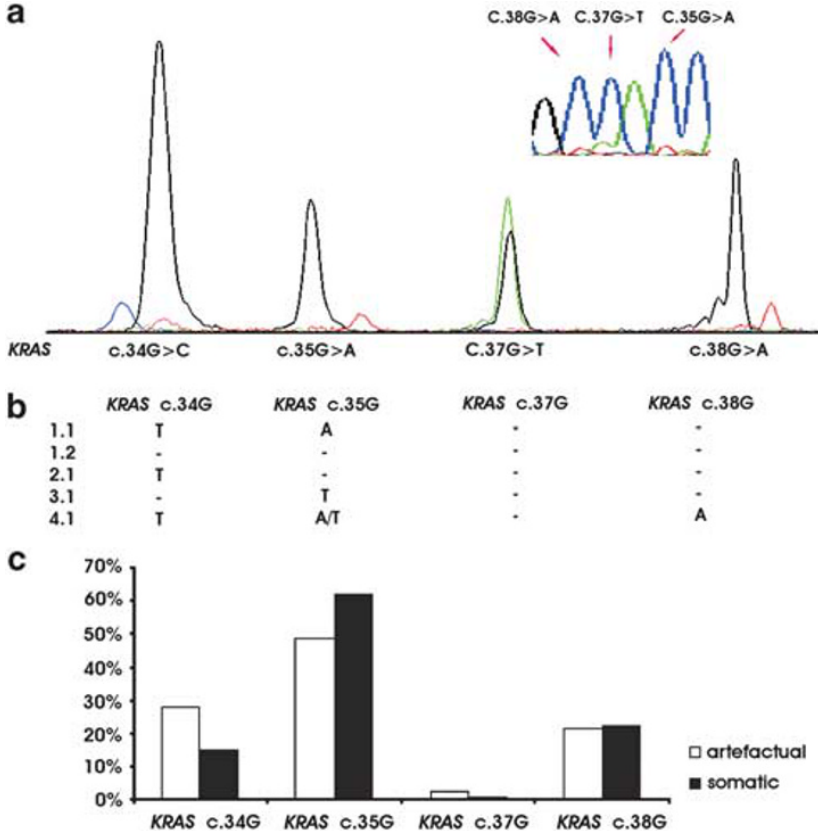

Figure $3 K R A S$ artefactual mutations in formalin-fixed paraffinembedded samples. (a) KRAS c.34G >C, KRAS c.35G > A, KRAS c.37G $>$ T, and KRAS c.38G $>$ A mutations observed from SNaPshot $\AA$ antisense pattern. KRAS c.35G $>$ A, KRAS c.37G $>$ T, and $K R A S$ c.38G > A mutations were also detected by direct sequencing from antisense primer (top right inset): fluorescent ddCTP incorporated during $\mathrm{SNaPshot} \AA$ or sequencing were different (black peak in SNaPshot $\AA$ pattern and blue peak in sequence pattern characterized wild-type complementary nucleotides). (b) Controversial KRAS genotypes obtained from distinct DNA extractions and PCR amplifications analyzing a formalin-fixed paraffin-embedded tumor sample. Dashes indicate KRAS wildtype nucleotide identity. The first digit in front of each sequence corresponds to the DNA extract number and the second to the amplification number. (c) Nucleotide distribution of somatic and artefactual mutations.

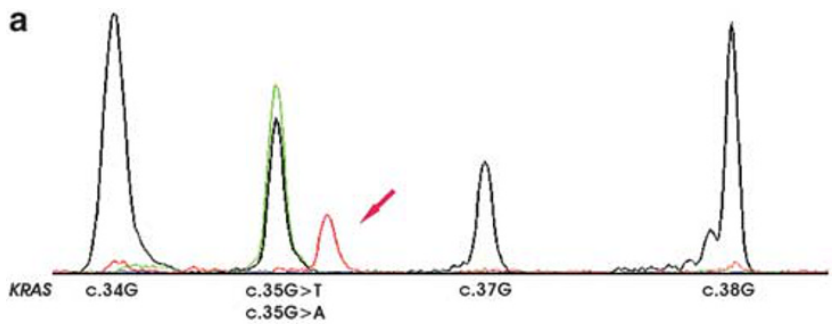

b

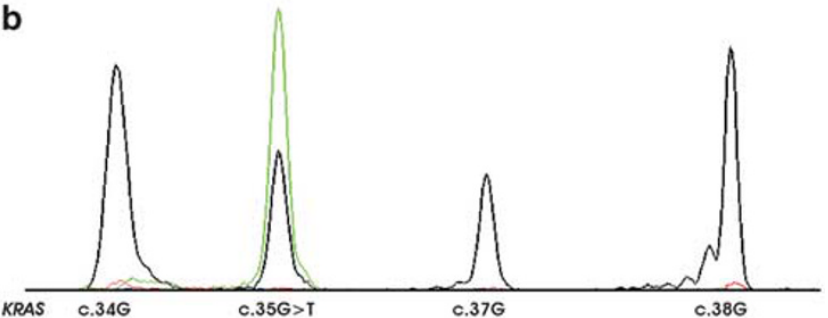

Figure 4 Effect of UNG treatment on KRAS artefactual mutations

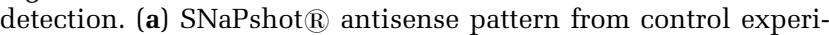
ment without UNG treatment under otherwise identical conditions detected KRAS c.35G $>\mathrm{A}$ (red arrow) and KRAS c.35G $>\mathrm{T}$ mutations. (b) After UNG treatment of the sample $K R A S$ c.35G $>$ A was not detected from the $\mathrm{SNaPshot} \AA$ antisense pattern.
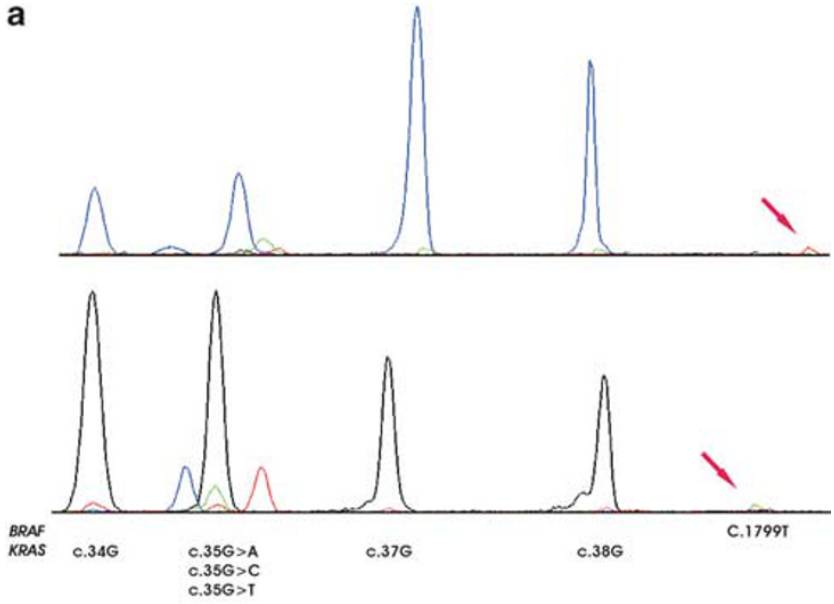

b
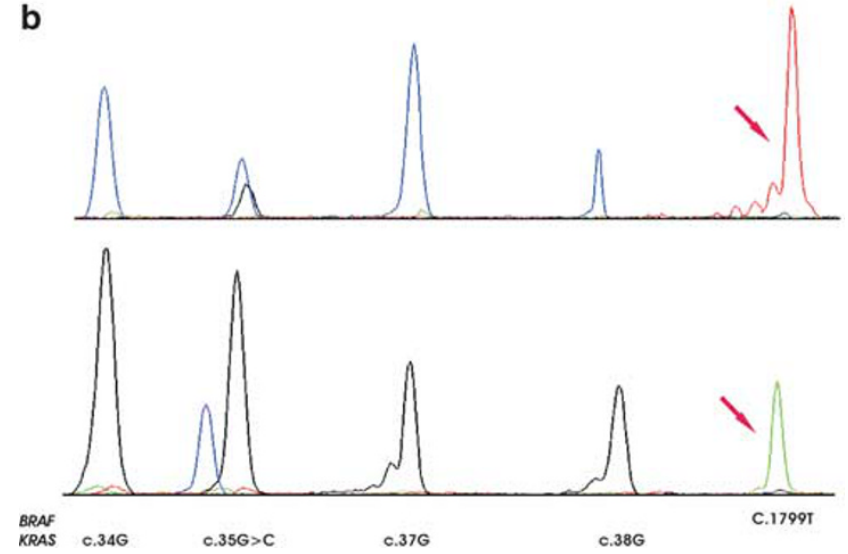

Figure 5 Effect of amplicon size on $\mathrm{SNaPshot} \mathbb{R}$ results. (a) SNaPshot $\AA$ sense and antisense patterns obtained from KRAS $173 \mathrm{bp} / B R A F 253 \mathrm{bp}$ amplification products: KRAS c. $35 \mathrm{G}>\mathrm{A}$, c. $35 \mathrm{G}>\mathrm{T}$, and c. $35 \mathrm{G}>\mathrm{C}$ mutations were detected. BRAF amplification product was almost undetectable (red arrow). (b) SNaP-

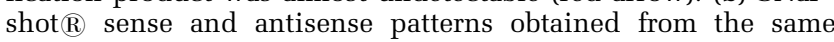
sample after KRAS $80 \mathrm{bp} / B R A F 126 \mathrm{bp}$ amplifications. KRAS c. $35 \mathrm{G}>\mathrm{A}$, c. $35 \mathrm{G}>\mathrm{T}$ mutations were no longer detected. $B R A F$ amplification product was detected as wild type (red arrow). This sample was considered KRAS c.35G >C mutated.

\section{Discussion}

We report the results of routine KRAS genotyping performed in a series of 1077 formalin-fixed paraffin-embedded tumor samples derived from patients with colorectal cancers, before anti-EGFR monoclonal antibodies treatment. KRAS mutations were found in $41.8 \%$ of the tumor samples that is in agreement with the previously estimated frequency of KRAS mutations in colorectal cancers. ${ }^{19,20}$ The three hot spots c. $35 \mathrm{G}>\mathrm{A}$, с. $38 \mathrm{G}>\mathrm{A}$, and c.35G $>$ T accounted for $77.5 \%$ of our KRAS mutated samples, which is also in agreement with previous reports. ${ }^{21,22}$

Even if BRAF p.V600E mutation involvement in resistance to anti-EGFR monoclonal antibodies treatment remains controversial, we decided to include its assessment in our KRAS mutational screening assay as its powerful negative prognostic factor role seems to be of importance. ${ }^{6-8}$ KRAS and 
$B R A F$ mutational status were simultaneously inves-

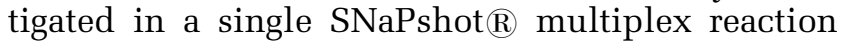
in 397 formalin-fixed paraffin-embedded tumor samples and $6.5 \%$ of the samples tested presented the BRAF p.V600E mutation in accordance with the frequencies reported in the literature. ${ }^{8}$ As expected, KRAS and BRAF mutations were found to be mutually exclusive. ${ }^{16}$ The fact that, in this large series, the frequencies, the distribution, and exclusiveness of KRAS and BRAF mutations are in total agreement with published studies validates our molecular analysis procedures. Furthermore, our KRAS genotyping assay was validated by different quality controls.

$K R A S$ activation is considered to be an early event in the colorectal adenoma-carcinoma sequence, suggesting that KRAS mutational status should be the same from primary tumors to metastases. Previous reports have demonstrated a reliable overall concordance between KRAS mutations in primary tumors and secondary localizations, but different KRAS genotypes could be observed in an average $10 \%$ of the paired samples analyzed. ${ }^{23,24}$ In our routine diagnostic procedure, $5(11.4 \%)$ of the 44 patients with paired primary tumors and metastases presented discordance in terms of KRAS mutational status. Recently, Baldus et $a l^{24}$ suggested that, to determine KRAS mutational status of colorectal cancer patients in clinical practice, primary tumors and distant metastases were more relevant tissue specimens than lymph-node metastases; their data indicated that different lymph-node metastases from a given tumor could differ in their KRAS genotype. We made the same observation in the present study: in patient 72 who presented a mutated KRAS primary tumor and a wild-type KRAS lymph node, further analyses detected the KRAS mutation in an additional lymph-node metastasis as well as in the distant liver metastasis.

Tumor heterogeneity had also been reported in primary colorectal carcinoma. Losi et $a{ }^{25}$ reported intratumor heterogeneity for KRAS mutations which is more common in early colorectal cancers $(60 \%)$ than in advanced colorectal cancers $(20 \%)$. In the study performed by Baldus et $a{ }^{24} 8 \%$ of paired tumor centre/invasive front samples from 100 patients with colorectal cancers presented different KRAS genotypes. This observation is of particular importance, since, in most laboratories, KRAS genotyping is usually performed on only one single tumor sample. In the present series, seven patients presented different KRAS genotypes within multiple synchronous primary tumor sites (multifocal cancer localization). These raised the question of the most relevant tumor localization that should be tested. In such circumstances, considering that metastases will most likely derive from the tumor with higher grade or stage, as illustrated by the case of patient 98 presented in this series, it may be reasonable to perform genotyping on the tumor with higher grade or stage. Considering our few discrepant results between primary tumors and metastases and within primary tumors between multifocal localization, it would be more relevant, in so far as anti-EGFR monoclonal antibodies treat metastatic disease, to perform KRAS genotyping on metastases. Moreover, considering the difficulty in obtaining metastatic tissue, at least in certain patients, analyses on blood samples should be of significant clinical interest. ${ }^{26}$

Our study illustrates that different mechanisms can lead to artefactual mutations, as previously reported. ${ }^{13,17,27}$ First, these artefacts have been reported when PCR amplification is performed on low amounts of DNA: a nucleotide misincorporation occurring during the first cycle of PCR will then be homogeneously carried by all PCR molecules generated during subsequent cycles. ${ }^{27}$ To reduce such artefacts, amplification starting from a minimum of $1 \mu \mathrm{g}$ of formalin-fixed paraffin-embedded recovered DNA and assessment of each mutation discovered from two independent amplification products is recommended. ${ }^{13}$ In the present study, each mutation found was confirmed by a second totally independent analysis (from new paraffin block sections to the final primer extended results). Nevertheless, 49 $(26.2 \%)$ of our non-contributive analyses appeared after using $1 \mu \mathrm{g}$ of formalin-fixed paraffin-embedded recovered DNA, suggesting that the observed artefactual mutations were not due to an insufficient quantity of DNA. The second mechanism able to generate artefactual mutations corresponds to chemical modification of DNA. Chemical modifications, affecting cytosine and guanine residues (which are KRAS nucleotide mutational hot spot) and resulting in $\mathrm{G}>\mathrm{A}$ transitions due to nucleotide misincorporations by DNA polymerase during amplification, have been reported during analysis of ancient DNA and formalin-fixed paraffin-embedded materials. ${ }^{13,17,28}$ Cytosine deamination, generating an uracil residue which leads to the incorrect insertion of an adenine residue during PCR amplification, is the predominant cause of such misincorporation and has been shown to generate artefactual EGFR mutation in formalin-fixed paraffin-embedded lung cancer samples. ${ }^{13,28}$ To explore the contribution of this mechanism to the $\mathrm{G}>\mathrm{A}$ artefactual transitions that we observed in this series, samples were treated with UNG which removes uracil from DNA, creating a strand break and thus is expected to lead to the disappearance of $\mathrm{G}>\mathrm{A}$ transition. We found that this treatment corrected only one artefactual result, suggesting that cytosine deamination is not the main mechanism of DNA template alterations observed in formalin-fixed paraffin-embedded samples. This is not surprising considering that in the studies reporting cytosine deamination in template DNA, artefactual mutations correspond only to $\mathrm{C} / \mathrm{G}$ to $\mathrm{T} / \mathrm{A}$ changes. In contrast, in our study, if $\mathrm{G}>\mathrm{A}$ transitions corresponded to the majority of the observed artefactual mutations 
(52.3\%), G>T and $\mathrm{G}>\mathrm{C}$ transversions were also observed at significant frequencies (36.4 and $11.3 \%$, respectively). KRAS $\mathrm{G}>\mathrm{A}$ transitions could also be ascribed to alterations affecting guanine residues, such as deamination leading to xanthine residue. However, when templates containing xanthine residues were used in PCRs, thymine misincorporation occurred in a minority of cases and depended on the Taq polymerases that were used. ${ }^{28}$ Finally, chemical modifications could also explain the artefactual $\mathrm{G}>\mathrm{T}$ transversions since in vivo, guanine residues are prone to oxidation leading to 8-hydroxyguanine which yields guanine to thymine transversion. $^{29}$ Other mechanisms may cause $\mathrm{G}>\mathrm{A}$ transitions and $\mathrm{G}>\mathrm{T}$ transversions. One such mechanism results from the tendency for Taq polymerase to add deoxyadenosine residues when it reaches the end of templates. This mechanism has been shown to cause substitutions when degraded templates that cause recombination between different template molecules are used in PCR: "jumping or template switching'. ${ }^{18}$ This phenomenon is expected to occur particularly frequently in nucleotide facing deoxycytidine residues. ${ }^{30}$ In our series, we found that the prominent mechanism leading to artefactual results was the well-known DNA fragmentation occurring during the formalin fixation and paraffin embedding processing. ${ }^{31}$ Indeed, electrophoresis of our formalin-fixed paraffin-embedded DNA samples clearly shows this phenomenon (Figure 6) and we observed artefactual results on DNA yielding no amplification or weak amplification, when a BRAF $253 \mathrm{bp}$ genomic segment was PCR amplified. Furthermore, the reduction of the KRAS amplicon size suppressed the artefactual KRAS mutations initially observed in 12 samples. As previously documented, DNA fragmentation

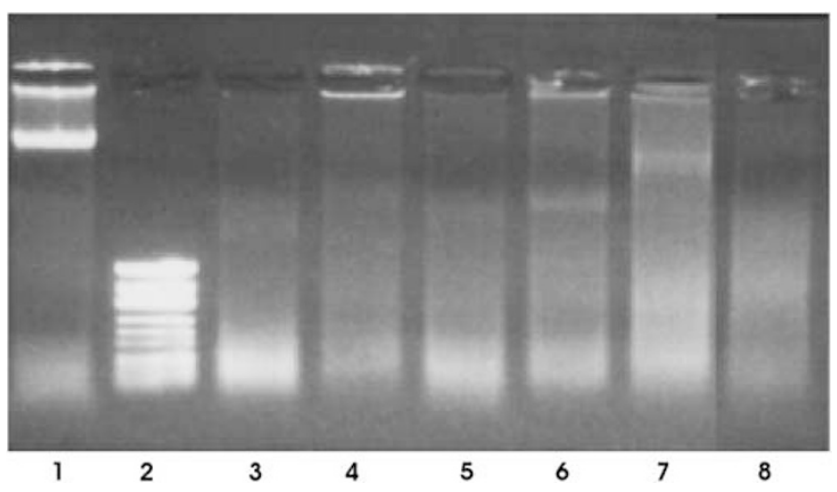

Figure 6 DNA fragmentation occurring in formalin-fixed paraffinembedded tissue samples. In all, $1 \mu \mathrm{g}$ of genomic DNA extracted from frozen or formalin-fixed paraffin-embedded tissues was run on a $1 \%$ agarose gel containing ethidium bromide. A single high molecular weight band was observed for DNA isolated from frozen tissue (lane 1) whereas low molecular weight smears were observed for DNA isolated from formalin-fixed paraffin-embedded tissue samples. The smear seamed of higher molecular weight for KRAS/BRAF contributive DNA sample (lane 8) compared with KRAS/BRAF non-contributive DNA samples (lanes 3-7). Lane 2 corresponds to the $\phi \times 174$ DNA Hinf I molecular size standard (24-726 bp). leads to intermolecular jumping events on both sense and antisense DNA strands during the PCR amplification and then respectively to $G>A$ and $\mathrm{G}>\mathrm{T}$ artefactual substitutions. ${ }^{18}$

Finally, we found that $67 \%$ of the artefactual results were obtained from formalin-fixed paraffinembedded tumor samples addressed by pathology laboratories situated in high humidity and temperature level climates. These atmospheric conditions could result in poor dehydration of the tumor samples before paraffin embedding. Hydrolysis of DNA glycosyl bonds leading to depurination (guanine and adenine liberation) has been described in vivo. ${ }^{29}$ At the site of base loss, the DNA chain is weakened and undergoes cleavage by a $\beta$-elimination process within a few days. However, in living cells, a DNA repair process is rapidly initiated to prevent such depurination. Conversely, it can be predicted that if deprived of the repair mechanisms provided in living cells, hydrated DNA is spontaneously degraded to short fragments, a phenomenon that we frequently observed. Therefore, it is likely that an insufficient dehydration of samples will lead to DNA fragmentation. Fungi development on formalin-fixed paraffin-embedded blocks observed by pathologists during storage is an argument favoring such a hypothesis. Furthermore, considering that the most important route for hydrated DNA is depurination, cleavage occurring at KRAS guanine mutation hot spots and intermolecular jumping events at these breakpoints could explain the majority of $\mathrm{G}>\mathrm{A}$ artefactual transitions we observed.

\section{Conclusion}

Molecular pathology is the natural evolution of anatomical pathology. In contrast to immunohistochemistry that has not radically changed pathology processing, molecular pathology needs more fundamental technical adjustments. It is important to emphasize that histopathological methods (ie, fixation, paraffin embedding and block sectioning) currently used and performed in pathology laboratories have been developed and designed for morphological studies. It is in a way surprising (and a very good thing) that nucleic acids are so far fairly well preserved by such drastic methods. Nevertheless, this fact should not exclude the development of quality control schemes in molecular pathology. The artefactual KRAS and $B R A F$ mutations due to fixation and storage conditions described in our study reinforced this change of paradigm in our medical practice, especially in the pre-analytical phases: (1) by using extra precaution from tissue sampling to block sectioning in order to avoid tissue cross-contamination, (2) by using more standardized fixative reagent (the quest for the perfect fixative solution with high quality morphological results, protein and nucleic acid preservation 
is still underway ...) to avoid DNA fragmentation, (3) by performing morphological examination (by a trained pathologist) of every tumor screened for somatic mutation in order to estimate the percentage of tumor cells in the sample tested, (4) by using controlled storage conditions to avoid nucleic acid hydrolysis, (5) by using adapted primers and short size amplicons, and (6) by controlling every mutation found with double techniques. With these conditions, we will be able to achieve routine molecular pathology with the high quality and security standards that we need to offer our patients.

\section{Acknowledgements}

The 'Laboratoire de Génétique Somatique des Tumeurs' is part of the platform de Génétique des Tumeurs de Haute-Normandie labelled and funded by the French Institut National du Cancer (INCa) to perform molecular analyses in cancer.

\section{Disclosure/conflict of interest}

The authors declare no conflict of interest.

\section{References}

1 Yarden Y, Sliwkowski MX. Untangling the ErbB signalling network. Nat Rev Mol Cell Biol 2001;2: 127-137.

2 Lièvre A, Bachet JB, Le Corre D, et al. KRAS mutation status is predictive of response to cetuximab therapy in colorectal cancer. Cancer Res 2006;66:3992-3995.

3 Di Fiore F, Blanchard F, Charbonnier F, et al. Clinical relevance of KRAS mutation detection in metastatic colorectal cancer treated by cetuximab plus chemotherapy. Br J Cancer 2007;96:1166-1169.

4 Amado RG, Wolf M, Peeters M, et al. Wild-type KRAS is required for panitumumab efficacy in patients with metastatic colorectal cancer. J Clin Oncol 2008;26: 1626-1634

5 Garnett MJ, Marais R. Guilty as charged: B-RAF is a human oncogene. Cancer Cell 2004;6:313-319.

6 Di Nicolantonio F, Martini M, Molinari F, et al. Wildtype BRAF is required for response to panitumumab or cetuximab in metastatic colorectal cancer. J Clin Oncol 2008;26:5705-5712.

7 Tol J, Nagtegaal ID, Punt CJ. BRAF mutation in metastatic colorectal cancer. N Engl J Med 2009; 361:98-99.

8 Van Cutsem E, Lang I, Folprecht G, et al. Cetuximab plus FOLFIRI in the treatment of metastatic colorectal cancer (mCRCs): The influence of KRAS and BRAF biomarkers on outcome: Updated data from the CRYSTAL trial. Abstract No: 281. ASCO GI 2010).

9 Erbitux European public assessment report. EMEA/ 327144/2009, EMEA/H/C/558. European Medicines Agency 2009.

10 Vectibix European public assessment report. EMEA/H/ C/741. European Medicines Agency 2007.

11 Van Krieken JH, Jung A, Kirchner T, et al. KRAS mutation testing for predicting response to anti-EGFR therapy for colorectal carcinoma: proposal for an
European quality assurance program. Virchows Arch 2008;453:417-431.

12 Perspectives on Emerging Technology (POET). Report: KRAS Mutation Testing for Colorectal Cancer (CRCs). College of American Pathologists: Northfield, IL, 2009.

13 Marchetti A, Felicioni L, Buttitta F. Assessing EGFR mutations [author reply]. N Engl J Med 2006;354: 526-528.

14 Williams C, Pontén F, Moberg C, et al. A high frequency of sequence alterations is due to formalin fixation of archival specimens. Am J Pathol 1999; 155:1467-1471.

15 Van Oers JM, Lurkin I, van Exsel AJ, et al. A simple and fast method for the simultaneous detection of nine fibroblast growth factor receptor 3 mutations in bladder cancer and voided urine. Clin Cancer Res 2005;11:7743-7748.

16 Rajagopalan H, Bardelli A, Lengauer C, Kinzler KW, Vogelstein B, Velculescu VE. Tumorigenesis: RAF/RAS oncogenes and mismatch-repair status. Nature 2002;418:934.

17 Hofreiter M, Jaenicke V, Serre D, Haeseler Av A, Pääbo S. DNA sequences from multiple amplifications reveal artifacts induced by cytosine deamination in ancient DNA. Nucleic Acids Res 2001;29:4793-4799.

18 Pääbo S, Irwin DM, Wilson AC. DNA damage promotes jumping between templates during enzymatic amplification. J Biol Chem 1990;265:4718-4721.

19 Andreyev HJ, Norman AR, Cunningham D, Oates JR, Clarke PA. Kirsten ras mutations in patients with colorectal cancer: the multicenter 'RASCAL' study. J Natl Cancer Inst 1998;90:675-684.

20 Andreyev HJ, Norman AR, Cunningham D, et al. Kirsten ras mutations in patients with colorectal cancer: the 'RASCAL II' study. Br J Cancer 2001;85: 692-696.

21 Neumann J, Zeindl-Eberhart E, Kirchner T, Jung A. Frequency and type of KRAS mutations in routine diagnostic analyses of metastatic colorectal cancer. Pathol Res Pract 2009;205:858-862.

22 Weichert W, Schewe C, Lehmann A, et al. KRAS genotyping of paraffin-embedded colorectal cancer tissue in routine diagnostics: comparison of methods and impact of histology. J Mol Diagn 2010;12:35-42.

23 Artale S, Sartore-Bianchi A, Veronese SM, et al. Mutations of KRAS and BRAF in primary and matched metastatic sites of colorectal cancer. J Clin Oncol 2008;26:4217-4219.

24 Baldus SE, Schaefer KL, Engers R, Hartleb D, Stoecklein NH, Gabbert HE. Prevalence and heterogeneity of KRAS, BRAF, and PIK3CA mutations in primary colorectal adenocarcinomas and their corresponding metastasis. Clin Cancer Res 2010;16:790-799.

25 Losi L, Baisse B, Bouzourene H, Benhattar J. Evolution of intratumoral genetic heterogeneity during colorectal cancer progression. Carcinogenesis 2005;26:916-922.

26 Di Fiore F, Charbonnier F, Lefebure B, et al. Clinical interest of KRAS mutation detection in blood for antiEGFR therapies in metastatic colorectal cancer. Br J Cancer 2008;99:551-552.

27 Akbari M, Hansen MD, Halgunset J, Skorpen F, Krokan HE. Low copy number DNA template can render polymerase chain reaction error prone in a sequencedependent manner. J Mol Diagn 2005;7:36-39.

28 Stiller M, Green RE, Ronan M, et al. Patterns of nucleotide misincorporations during enzymatic 
amplification and direct large-scale sequencing of ancient DNA. Proc Natl Acad Sci USA 2006;103: 13578-13584.

29 Lindahl T. Instability and decay of the primary structure of DNA [Review]. Nature 1993;362:709-715.

30 Kwok S, Kellogg DE, McKinney N, et al. Effects of primer-template mismatches on the polymerase chain reaction: human immunodeficiency virus type 1 model studies. Nucleic Acids Res 1990;18:999-1005.

31 Inoue $\mathrm{T}$, Nabeshima K, Kataoka H, Koono M. Feasibility of archival non-buffered formalin-fixed and paraffin-embedded tissues for PCR amplification: an analysis of resected gastric carcinoma. Pathol Int 1996;46:997-1004. 